\title{
Relaxation times for chiral transport phenomena and spin polarization in a strongly coupled plasma
}

\author{
Shiyong $\mathrm{Li}^{*}$ and Ho-Ung Yee \\ Department of Physics, University of Illinois, Chicago, Illinois 60607, USA
}

(Received 7 June 2018; published 21 September 2018)

\begin{abstract}
We compute the dynamical relaxation times for chiral transport phenomena in a strongly coupled regime using the AdS/CFT correspondence. These relaxation times can be a useful proxy for the dynamical timescale for achieving equilibrium spin polarization of quasiparticles in the presence of a magnetic field and fluid vorticity. We identify the Kubo relations for these relaxation times and clarify some previous issues regarding time dependence of the chiral vortical effect. We study the consequences of imposing timereversal invariance on parity-odd thermal noise fluctuations that are related to chiral transport coefficients by the fluctuation-dissipation relation. We find that time-reversal invariance dictates the equality between some of the chiral transport coefficients as well as their relaxation times.
\end{abstract}

DOI: 10.1103/PhysRevD.98.056018

\section{INTRODUCTION}

The anomalous transport phenomena arising from the chiral anomaly that could be present in the chiral-symmetry restored phase of quark-gluon plasma created in ultrarelativistic heavy-ion collisions have attracted much attention recently (see the reviews [1-6] and the references therein). One such phenomenon, the chiral magnetic effect (CME) [7,8], predicts a dipole charge separation [9-12] which is proportional to the magnetic field produced in offcentral collisions along the direction perpendicular to the reaction plane [13-18], that could result in the observable charge dependence of angular correlations of two charged pions [19-23]. A closely related phenomenon, the chiral vortical effect (CVE) [24,25], may also induce additional separation of charged hadrons proportional to the vorticity or the kinetic angular momentum of the plasma fireball $[26,27]$. Both of these effects rely on the fluctuating chiral charge (or axial charge) imbalance between the righthanded and the left-handed quarks that could arise either from initial state fluctuations of color charge density [28] or from statistical sphaleron transitions in the quark-gluon plasma [29,30]. These effects also induce interesting gapless propagating modes of chiral charges, the chiral magnetic wave [31,32], which may induce a charge quadrupole moment proportional to the magnetic field

\footnotetext{
sli72@uic.edu

†hyee@uic.edu
}

Published by the American Physical Society under the terms of the Creative Commons Attribution 4.0 International license. Further distribution of this work must maintain attribution to the author(s) and the published article's title, journal citation, and DOI. Funded by SCOAP.
[33-35]. To clearly disentangle the proposed signatures of these effects from the other sources that constitute the background [36-40], the isobar heavy-ion collision experiments comparing $\mathrm{Rb}$ and $\mathrm{Zr}$ that have a $10 \%$ difference in the strength of the magnetic field are currently ongoing in the Relativistic Heavy-Ion Collider at the Brookhaven National Laboratory [3,41]. The evidence for the CME has been observed recently in the condensed matter systems of Dirac [42] and Weyl $[43,44]$ semimetals where quasielectrons feature similar concepts of chirality found in relativistic massless fermions.

In the limit of static and homogeneous magnetic field and vorticity, the magnitude of the CME and the CVE conductivities defined by

$$
\begin{aligned}
J_{\text {chiral }}^{\mu} & =\sigma_{B} B^{\mu}+\sigma_{V} \omega^{\mu}, \quad B^{\mu} \equiv \frac{1}{2} \epsilon^{\mu \nu \alpha \beta} u_{\nu} F_{\alpha \beta}, \\
\omega^{\mu} & \equiv \epsilon^{\mu \nu \alpha \beta} u_{\nu} \nabla_{\alpha} u_{\beta}
\end{aligned}
$$

is completely fixed by the chiral anomaly. For the simplest case of a theory with one right-handed chiral fermion species, we have

$$
\sigma_{B}=\frac{\mu}{4 \pi^{2}}, \quad \sigma_{V}=\frac{\mu^{2}}{8 \pi^{2}},
$$

where $\mu$ is $\mu_{R}$, the (chiral) chemical potential; we have dropped the $\mathrm{R}$ to simplify our notation from here and

\footnotetext{
${ }^{1}$ For QCD with $N_{F}$ light flavors of Dirac quarks, the net current is a sum of left- and right-handed currents, both of which should be multiplied by $N_{c} N_{F}$, and the chemical potentials are the chiral ones, $\mu_{L / R}=\mu_{V} \mp \mu_{A}$. The effects for the left-handed currents carry the additional opposite sign compared to those for the right-handed currents.
} 
throughout the paper. For $\sigma_{V}$, there exists an additional correction of order $T^{2}$, where $T$ is temperature, that is related to the mixed chiral-gravitational anomaly $[45,46]$. These results are topologically protected and not modified by interactions, which has been confirmed in many different approaches, for instance, in weakly coupled perturbation theory [47-49], the AdS/CFT correspondence [50-55], hydrodynamics with the second law of thermodynamics [56-58], effective field theories [59-61], and the chiral kinetic theory [62-72].

In out-of-equilibrium conditions, these conductivities depend more on microscopic interactions and generally deviate from their equilibrium values. The question of particular interests is how these conductivities behave when the magnetic field and the vorticity is time dependent with a frequency $\omega$ :

$$
J_{\text {chiral }}^{\mu}(\omega)=\sigma_{B}(\omega) B^{\mu}(\omega)+\sigma_{V}(\omega) \omega^{\mu}(\omega),
$$

where $\mu$ runs over all four spacetime directions. The computation of the frequency dependence of the CME has been done both in weakly coupled $[47,65,73]$ and strongly coupled regimes $[50,74,75]$, and the results are not universal anymore. In the quasiparticle picture of these effects at weak coupling [73], the results at finite frequency depend on the relaxation dynamics of spin polarization of chiral fermions along the direction of magnetic field or vorticity, in addition to the more topological effect from the Berry phase that is responsible for the equilibrium values [62-64]. For example, there appears a new contribution to the current from spin magnetization at finite frequency [73].

Another way of looking at this is that the frequencymomentum dependence of chiral conductivities is related to the parity (P)-odd thermal noise fluctuations via the fluctuation-dissipation theorem [76]. We will review this connection in more detail in Sec. III, where we derive the general consequences on these chiral conductivities when we impose the time-reversal (T) invariance of the theory on the P-odd thermal noise fluctuations. ${ }^{2}$ In the simplest case of current-current noise correlation, one derives the fluctuation-dissipation relation

$$
\left\langle\delta \boldsymbol{J}^{i}(k) \delta \boldsymbol{J}^{j}(-k)\right\rangle \equiv G_{r r}^{i j}(k)=\left(\frac{1}{2}+n_{B}(\omega)\right) \rho^{i j}(k),
$$

in terms of the spectral density $\rho^{i j}(k) \equiv G_{r a}^{i j}(k)-G_{a r}^{i j}(k)=$ $G_{r a}^{i j}(k)+\left(G_{r a}^{j i}(k)\right)^{*}$, where $k$ is the four momentum $(\omega, \vec{k})$; $i, j$ run only spatial indices; and $n_{B}(\omega)$ is the Bose-Einstein distribution function. The last equality in Eq. (1.4) is a consequence of the Hermiticity of current operators (that is

\footnotetext{
${ }^{2}$ Note that a right-handed Weyl fermion remains right handed under time-reversal transformation since helicity, which is a product of momentum and spin, is $\mathrm{T}$ even. Without a $\mathrm{CP}$ violating complex phase, a chiral (gauge) theory is invariant under time reversal and $C P$.
}

equivalent to the real-valuedness of the retarded correlation function in coordinate space) and the translational invariance of the system. Note that $\rho^{i j}(k)$ is a Hermitian matrix in $(i, j)$ but need not be real valued. This Hermiticity of $\rho^{i j}(k)$ together with translational invariance is sufficient to guarantee that the noise correlation is real valued in coordinate space as it should be. Recalling that the retarded correlation function is $G_{R}^{i j}(k)=-i G_{r a}^{i j}(k)$, the P-odd part of the retarded current correlation function

$$
G_{R}^{i j}(k)=i \tilde{\sigma}_{B}(k) \epsilon^{i j l} \boldsymbol{k}^{l}
$$

that is responsible for frequency-momentum dependent chiral magnetic effect, ${ }^{3}$ gives a P-odd contribution to the spectral density [76]

$$
\rho^{i j}(k) \sim-2 \operatorname{Im}\left[\tilde{\sigma}_{B}(k)\right] i \epsilon^{i j l} \boldsymbol{k}^{l} \equiv \rho^{\mathrm{odd}}(k) i \epsilon^{i j l} \boldsymbol{k}^{l},
$$

in terms of a real-valued function $\rho^{\text {odd }}(k)=-2 \operatorname{Im}\left[\tilde{\sigma}_{B}(k)\right]$. The reality of the retarded correlation function in coordinate space gives a constraint $\tilde{\sigma}_{B}(k)=\left(\tilde{\sigma}_{B}(-k)\right)^{*}$, which dictates that

$$
\rho^{\text {odd }}(-k)=-\rho^{\text {odd }}(k),
$$

and especially the P-odd spectral density vanishes in the zero momentum limit. When $\boldsymbol{k} \rightarrow 0$, we have $\rho^{\text {odd }}(\omega)=$ $2 \xi_{5} \omega+\cdots$ in the small $\omega$ limit, where the transport coefficient $\xi_{5}$ roughly corresponds to a second-order correction to the CME $[57,77]{ }^{4}$

$$
J_{C M E}^{\mu}=\sigma_{B} B^{\mu}+\xi_{5}(u \cdot \nabla) B^{\mu},
$$

and together with $n_{B}(\omega) \rightarrow \frac{T}{\omega}$ in (1.4) in $\omega \ll T$, we have the P-odd thermal noise fluctuations in the hydrodynamics regime,

$$
\left\langle\delta \boldsymbol{J}^{i}(\boldsymbol{x}, t) \delta \boldsymbol{J}^{j}\left(\boldsymbol{x}^{\prime}, t^{\prime}\right)\right\rangle=2 T \xi_{5} \epsilon^{i j k} \frac{\partial}{\partial \boldsymbol{x}^{k}} \delta\left(\boldsymbol{x}-\boldsymbol{x}^{\prime}\right) \delta\left(t-t^{\prime}\right) .
$$

Since the thermal noise spectrum in general should depend on real-time microscopic dynamics of the thermal ensemble, they cannot be topological and should be nonuniversal. This is consistent with the fact that the above P-odd spectral density is insensitive to the topologically protected value of $\lim _{k \rightarrow 0} \lim _{\omega \rightarrow 0} \operatorname{Re}\left[\tilde{\sigma}_{B}(k)\right]=\frac{\mu}{4 \pi^{2}}$ and only depends on the nonuniversal part of $\operatorname{Im}\left[\tilde{\sigma}_{B}(k)\right]$. The $\xi_{5}$ was computed in two-flavor perturbative QCD in the leading $\log$ as [48]

\footnotetext{
${ }^{3}$ As will be seen in Sec. II, this $\tilde{\sigma}_{B}(k)$, although related, is not precisely equal to the conductivity $\sigma_{B}(\omega)$ appearing in the constitutive relation (1.3) due to the energy-momentum sheardiffusion pole.

${ }^{4}$ This is not precise. The more precise such "Kubo relation" will be derived in Sec. II.
} 


$$
\xi_{5} \approx-\frac{0.5}{\alpha_{s}^{2} \log \left(1 / \alpha_{s}\right)} \frac{\sigma_{B}}{T} .
$$

These frequency-dependent chiral conductivities are also important in any realistic simulation of heavy-ion collisions in an attempt to quantify the effects from these anomalous transport phenomena on the experimental observables $[11,12]$.

A useful characterization of frequency-dependent transport coefficients is the relaxation time defined by

$$
\sigma(\omega)=\sigma(0)(1+i \omega \tau)+\mathcal{O}\left(\omega^{2}\right),
$$

which is motivated from a simple relaxation time pole expanded in first order in $\omega$,

$$
\sigma(\omega)=\sigma(0) \frac{i / \tau}{\omega+i / \tau},
$$

or equivalently, from an Israel-Stewart-type treatment expanded in first order in time derivative $u \cdot \nabla$,

$$
\begin{aligned}
(u \cdot \nabla) J_{C M E}^{\mu} & =-\frac{1}{\tau_{B}}\left(J_{C M E}^{\mu}-\sigma_{B} B^{\mu}\right) \rightarrow \\
J_{C M E}^{\mu} & =\sigma_{B} B^{\mu}-\sigma_{B} \tau_{B}(u \cdot \nabla) B^{\mu}+\cdots .
\end{aligned}
$$

We should note however that the real response of the system may not be simply given by the Israel-Stewart form [78], and in that case the relaxation time defined as above should instead be viewed as a characteristic dynamical timescale of the problem that appears in second-order hydrodynamics [79].

The full anomalous transport phenomena in the anomaly frame $[58,80,81]$ or no-drag frame $[82,83]$ consist of four transport coefficients in the leading order of derivatives:

$T_{\text {chiral }}^{\mu \nu}=\sigma_{\epsilon}^{B}\left(B^{\mu} u^{\nu}+B^{\nu} u^{\mu}\right)+\sigma_{\epsilon}^{V}\left(\omega^{\mu} u^{\nu}+\omega^{\nu} u^{\mu}\right)$,

$J_{\text {chiral }}^{\mu}=\sigma_{B} B^{\mu}+\sigma_{V} \omega^{\mu}$.

The two additional transport coefficients, $\sigma_{\epsilon}^{B / V}$, are responsible for the anomalous energy flow or momentum density along the magnetic field and vorticity. Their values are also fixed by chiral anomaly to be (up to temperature corrections) $[80,81]$

$$
\sigma_{\epsilon}^{B}=\frac{\mu^{2}}{8 \pi^{2}}, \quad \sigma_{\epsilon}^{V}=\frac{\mu^{3}}{6 \pi^{2}} .
$$

We correspondingly introduce the four relaxation times in the next order in the time derivative ${ }^{5}$ :

\footnotetext{
${ }^{5}$ Using the equation of motion of ideal hydrodynamics and the Bianchi identity for the field strength tensor, one can replace the time derivative $u \cdot \nabla$ with spatial derivatives. But, this is equivalent up to equations of motion, and does not affect the Kubo relations derived from the equation of motion.
}

$$
\begin{aligned}
T_{\text {chiral }}^{\mu \nu}= & \sigma_{\epsilon}^{B}\left(B^{\mu}-\tau_{\epsilon}^{B}(u \cdot \nabla) B^{\mu}\right) u^{\nu}+\sigma_{\epsilon}^{V}\left(\omega^{\mu}-\tau_{\epsilon}^{V}(u \cdot \nabla) \omega^{\mu}\right) u^{\nu} \\
& +(\mu \leftrightarrow \nu), \\
J_{\text {chiral }}^{\mu}= & \sigma_{B}\left(B^{\mu}-\tau_{B}(u \cdot \nabla) B^{\mu}\right)+\sigma_{V}\left(\omega^{\mu}-\tau_{V}(u \cdot \nabla) \omega^{\mu}\right) .
\end{aligned}
$$

In this work, we compute these relaxation times, $\left(\tau_{\epsilon}^{B / V}\right.$, $\left.\tau_{B / V}\right)$, in the strongly coupled regime using the AdS/CFT correspondence. The $\tau_{B}$ has previously been worked out in Ref. [54].

The dynamics of spin polarization that underlies the above relaxation times is more general beyond the massless chiral limit and is applicable even for massive spinful particles such as the Lambda baryon. In the theoretical understanding of recently measured $\Lambda$ hyperon spin polarization in off-central heavy-ion collisions at the RHIC [84], it is vitally important to know the dynamical timescale of this spin polarization, that is, the relaxation time it takes to achieve the spins polarized in a finite fluid vorticity or magnetic field. Therefore, by studying the relaxation times of the anomalous chiral transport phenomena in the massless chiral limit, we can get a useful proxy for this important timescale at the quark level before the hadronization happens where the quark spin polarization is transferred to the $\Lambda$ hyperon spin polarization. This is another motivation we have in this study.

In Sec. III we show that the T invariance of a microscopic theory imposed on the P-odd thermal noise fluctuations of momenta and currents requires

$$
\sigma_{\epsilon}^{B}=\sigma_{V}, \quad \tau_{\epsilon}^{B}=\tau_{V} .
$$

The first equality has been observed before in several different approaches [80,81], but its connection to the $\mathrm{T}$ invariance seems novel. The second equality is our new finding. We confirm this in our numerical computation in the AdS/CFT correspondence within the numerical accuracy of our analysis. It would be interesting to connect this result with the positivity of entropy production.

The frequency dependence of the CVE was previously considered in Refs. [74,75] in both weakly and strongly coupled regimes. Writing the P-odd part of the retarded current-momentum correlation function as

$$
\left\langle J^{i}(k) T^{0 j}(-k)\right\rangle_{R}=i \tilde{\sigma}_{V}(k) \epsilon^{i j l} \boldsymbol{k}^{l},
$$

it was observed that $\tilde{\sigma}_{V}(k)$ in the hydrodynamic regime behaves as

$\tilde{\sigma}_{V}(k)=\left(\sigma_{V}-\frac{n}{\epsilon+p} \sigma_{\epsilon}^{V} \frac{\omega}{\omega+i \gamma_{\eta} \boldsymbol{k}^{2}}\right) \frac{i \gamma_{\eta} \boldsymbol{k}^{2}}{\omega+i \gamma_{\eta} \boldsymbol{k}^{2}}$,

where $n$ is the charge density and $\gamma_{\eta}=\eta /(\epsilon+p)$ is the shear-diffusion constant. The physics behind the appearance of the shear-diffusion pole structure was also explained in Ref. [75]: it is a consequence of the 
energy-momentum conservation Ward identity. From this expression, it was observed that the retarded correlation function $\tilde{\sigma}_{V}(k)$ vanishes in the $\boldsymbol{k} \rightarrow 0$ limit for all $\omega \neq 0$, while the strict $\omega=0$ limit reproduces the correct CVE coefficient $\sigma_{V}$.

There is nothing wrong in these observations, but we point out that this retarded correlation function, $\tilde{\sigma}_{V}(k)$, is not directly equal to the frequency-momentum dependent transport coefficient, $\sigma_{V}(k)$, appearing in the constitutive relations: $\tilde{\sigma}_{V}(k) \neq \sigma_{V}(k)$. One way of seeing why these observations are not related to $\sigma_{V}(\omega)$ is that (1.19) is derived from the constitutive relation at leading order, that is (1.14), without any relaxation time or higher-order corrections in the constitutive relations. To extract the relaxation time appearing in the transport coefficient $\sigma_{V}(\omega)$ from the retarded correlation function $\tilde{\sigma}_{V}(k)$, we need to work with the constitutive relations at next leading order (1.16) including the relaxation times to derive the correct Kubo relations between the relaxation time and the correlation function $\tilde{\sigma}_{V}(k)$. We perform this exercise in Sec. II.

Our paper is organized as follows. In Sec. II we derive the correct Kubo relations between the relaxation times of chiral transport phenomena and the retarded correlation functions of energy-momenta and currents. In Sec. III we study the consequences of imposing the time-reversal invariance on the P-odd thermal noise fluctuations that can be obtained from the retarded correlation functions via the fluctuation-dissipation relation: we find that $\mathrm{T}$ invariance dictates $\tau_{\epsilon}^{B}=\tau_{V}$. We then proceed in Sec. IV to the numerical computation of these relaxation times in the AdS/CFT correspondence. We conclude with discussions in Sec. V.

\section{KUBO RELATIONS FOR THE RELAXATION TIMES OF CHIRAL TRANSPORT PHENOMENA}

Hydrodynamics is an effective theory describing dynamics of a thermal system on large length scales and timescales. The relevant degrees of freedom are the local velocity $u^{\mu}(x)$ (satisfying $u \cdot u=-1$ ) and the local densities of conserved quantities such the energy $\epsilon(x)$ and a charge $n(x)$. In the presence of external background fields, the hydrodynamic equations take the following form,

$$
\nabla_{\mu} T^{\mu \nu}=F^{\nu \alpha} J_{\alpha}, \quad \nabla_{\mu} J^{\mu}=C E_{\mu} B^{\mu}, \quad C=\frac{1}{4 \pi^{2}},
$$

together with the constitutive relations which express $T^{\mu \nu}$ and $J^{\mu}$ in terms of the local hydrodynamics variables themselves. These constitutive relations are organized by the number of spatial derivatives involved. The lowest order is the ideal hydrodynamics, and the viscous corrections start to appear in the first order of derivatives. In the case of a chiral fluid, new terms of chiral transport phenomena proportional to fluid vorticity and external magnetic field must be present in the constitutive relations in order to ensure the second law of the thermodynamics $[56,58]$

$$
\begin{aligned}
T^{\mu \nu} & =(\epsilon+p) u^{\mu} u^{\nu}+p g^{\mu \nu}+\tau^{\mu \nu}+\tau_{\text {chiral }}^{\mu \nu}, \\
J^{\mu} & =n u^{\mu}+\nu^{\mu}+\nu_{\text {chiral }}^{\mu}
\end{aligned}
$$

where our metric is mostly positive; $p=p(\epsilon, n)$ is the pressure; and $n$ is the charge density. Also $\Delta^{\mu}=$ $\left(u^{\mu} u^{\nu}+g^{\mu \nu}\right) \nabla_{\nu} \equiv \Delta^{\mu \nu} \nabla_{\nu}$ is the spatially projected derivative in the local rest frame; $E^{\mu}=F^{\mu \nu} u_{\nu}, B^{\mu}=\frac{1}{2} \epsilon^{\mu \nu \alpha \beta} u_{\nu} F_{\alpha \beta}$ are the electromagnetic fields; and $\omega^{\mu}=\epsilon^{\mu \nu \alpha \beta} u_{\nu} \partial_{\alpha} u_{\beta}$ is the fluid vorticity. The viscous and chiral transport terms are

$$
\begin{aligned}
\tau^{\mu \nu}= & -\eta \Delta^{\mu \alpha} \Delta^{\nu \beta}\left(\nabla_{\alpha} u_{\beta}+\nabla_{\beta} u_{\alpha}-\frac{2}{3} g_{\alpha \beta} \nabla_{\lambda} u^{\lambda}\right) \\
& -\zeta \Delta^{\mu \nu} \nabla_{\lambda} u^{\lambda}, \\
\tau_{\text {chiral }}^{\mu \nu}= & \sigma_{\epsilon}^{B}\left(B^{\mu}-\tau_{\epsilon}^{B}(u \cdot \nabla) B^{\mu}\right) u^{\nu}+\sigma_{\epsilon}^{V}\left(\omega^{\mu}-\tau_{\epsilon}^{V}(u \cdot \nabla) \omega^{\mu}\right) u^{\nu} \\
& +(\mu \leftrightarrow \nu), \\
\nu^{\mu}= & -\sigma T \Delta^{\mu}(\mu / T)+\sigma E^{\mu}, \\
\nu_{\text {chiral }}^{\mu}= & \sigma_{B}\left(B^{\mu}-\tau_{B}(u \cdot \nabla) B^{\mu}\right)+\sigma_{V}\left(\omega^{\mu}-\tau_{V}(u \cdot \nabla) \omega^{\mu}\right)
\end{aligned}
$$

where we include only the relaxation time terms of our interest at the next leading order in derivatives. The $\sigma_{B} \tau_{B}$ is equivalent to one of the second-order transport coefficients $-\xi_{5}$ studied in Refs. [48,57]. The electric conductivity and charge diffusion terms proportional to the conductivity $\sigma$ will not affect our Kubo relations for the chiral transport coefficients.

In the "anomaly" [58,80,81] or "no-drag" [82] frame, the leading-order chiral transport coefficients are given by

$\sigma_{B}=C \mu, \quad \sigma_{V}=\sigma_{\epsilon}^{B}=\frac{1}{2} C \mu^{2}, \quad \sigma_{\epsilon}^{V}=\frac{2}{3} C \mu^{3}$,

up to temperature corrections which are related to the mixed chiral-gravitational anomaly [45,46,59]. One can also choose to work in the Landau frame [56] where one removes the chiral transports in the energy-momentum tensor by redefining the fluid velocity as

$$
\begin{aligned}
u^{\mu}= & u_{L F}^{\mu}-\frac{1}{\epsilon+p}\left(\sigma_{\epsilon}^{B}\left(B^{\mu}-\tau_{\epsilon}^{B}(u \cdot \nabla) B^{\mu}\right)\right. \\
& \left.+\sigma_{\epsilon}^{V}\left(\omega^{\mu}-\tau_{\epsilon}^{V}(u \cdot \nabla) \omega^{\mu}\right)\right) .
\end{aligned}
$$

Note that this upsets the naive counting of derivative expansions. This will then shift the transport coefficients $\sigma_{B / V}$ and $\tau_{B / V}$ appearing in $\nu_{\text {chiral }}^{\mu}$ in the Landau frame as $\sigma_{B / V}^{L F}=\sigma_{B / V}-\frac{n}{\epsilon+p} \sigma_{\epsilon}^{B / V}, \quad \tau_{B / V}^{L F}=\tau_{B / V}-\frac{n}{\epsilon+p} \tau_{\epsilon}^{B / V}$. 
Recall that these two "frames" (or any other frame) are different descriptions of the same system: a change of variables. Any final physics results, such as the retarded correlation functions of operators or the spectrum of thermal fluctuations, should be the same independent of the choice of frames, provided we used the correct relations (2.6) between the transport coefficients appearing in different frames. Note that the fluid velocity is not such a physics result: it is a descriptive variable. While we will work in the anomaly/no-drag frame in the following, the same retarded correlation functions and the Kubo relations are reproduced in the Landau frame as well. In checking this explicitly, it is necessary to keep the induced second-order term in the Landau frame coming from the shear viscosity term when we make the shift in fluid velocity (2.5):

$-\frac{\eta}{\epsilon+p} \Delta^{\mu \alpha} \Delta^{\nu \beta}\left(\sigma_{\epsilon}^{B} \nabla_{\alpha} B_{\beta}+\sigma_{\epsilon}^{V} \nabla_{\alpha} \omega_{\beta}+(\alpha \leftrightarrow \beta)-\right.$ trace $)$.
The necessity of this higher-order term in showing the equivalence between the two frames was observed in Ref. [85], and the basic reason is a violation of the derivative counting in (2.5).

To obtain the Kubo relations for these chiral transport coefficients [74,81], we consider small fluctuations of metric and gauge field, $\left(h_{\mu \nu}, A_{\mu}\right)$, in the background of the static equilibrium plasma in flat spacetime. Furthermore, we restrict ourselves to the effects that are linear in these fluctuations $h_{\mu \nu}$ and $A_{\mu}$. These fluctuations will drive the fluid away from equilibrium, which is described by the fluid velocity $u^{\mu}=\left(1, u^{i}(t, \mathbf{x})\right)$ (valid to linear order in $u^{i}$ ) as well as $\delta \epsilon$ and $\delta n$, that are determined by solving the hydrodynamics equation of motion. For our purpose, it is enough to switch on just $\left(h_{t x}(t, y), h_{t z}(t, y)\right)$ and $\left(A_{x}(t, y), A_{z}(t, y)\right)$ which depend only on $(t, y)$. In the frequency-momentum Fourier space of $e^{-i \omega t+i k y}$, the constitutive relations that are linear in the fluctuations become

$$
\begin{aligned}
T^{t t} & =\epsilon+\delta \epsilon, \\
T^{t i} & =(\epsilon+p) u^{i}+p h_{t i}-\sigma_{\epsilon}^{B}\left(1+i \omega \tau_{\epsilon}^{B}\right) \epsilon^{i j k} \partial_{j} A_{k}-\sigma_{\epsilon}^{V}\left(1+i \omega \tau_{\epsilon}^{V}\right) \epsilon^{i j k} \partial_{j}\left(u^{k}+h_{t k}\right), \\
T^{i j} & =\left(p+c_{s}^{2} \delta \epsilon\right) \delta^{i j}-\eta\left(\partial_{i} u^{j}+\partial_{j} u^{i}-\frac{2}{3} \delta_{i j} \partial_{k} u^{k}\right), \\
J^{i} & =n u^{i}-\sigma_{B}\left(1+i \omega \tau_{B}\right) \epsilon^{i j k} \partial_{j} A_{k}-\sigma_{V}\left(1+i \omega \tau_{V}\right) \epsilon^{i j k} \partial_{j}\left(u^{k}+h_{t k}\right),
\end{aligned}
$$

where $c_{s}$ is the speed of sound. For the sake of simplicity, we drop the bulk viscosity $\zeta$. The relevant hydrodynamic equations of motion from the energy-momentum conservation become

$$
\begin{aligned}
& \partial_{t} \delta \epsilon+\left(\epsilon_{0}+P_{0}\right) \partial_{i} u^{i}=0 \\
& (\epsilon+p) \partial_{t} u^{i}+c_{s}^{2} \partial_{i} \delta \epsilon-\eta\left(\partial^{2} u^{i}+\partial_{j} \partial_{i} u^{j}-\frac{2}{3} \partial_{i} \partial_{k} u^{k}\right)-\sigma_{\epsilon}^{B}\left(1+i \omega \tau_{\epsilon}^{B}\right) \epsilon^{i j k} \partial_{t} \partial_{j} A_{k}-\sigma_{\epsilon}^{V}\left(1+i \omega \tau_{\epsilon}^{V}\right) \epsilon^{i j k} \partial_{t} \partial_{j}\left(u^{k}+h_{t k}\right) \\
& \quad=-(\epsilon+p) \partial_{t} h_{t i}-n \partial_{t} A_{i},
\end{aligned}
$$

from which we can solve for the velocity, the energy density and the charge density fluctuations,

$$
\begin{aligned}
\delta \epsilon= & 0, \quad \delta n=0, \\
u^{x}= & \frac{-h_{t z} \sigma_{\epsilon}^{V}\left(1+i \omega \tau_{\epsilon}^{V}\right) i \omega k \frac{i \gamma_{\eta} \boldsymbol{k}^{2}}{\epsilon+p}+h_{t x} \omega\left(\omega+i \gamma_{\eta} \boldsymbol{k}^{2}\right)+A_{x} \omega \frac{n}{\epsilon+p}\left(\omega+i \gamma_{\eta} \boldsymbol{k}^{2}\right)}{-\left(\omega+i \gamma_{\eta} \boldsymbol{k}^{2}\right)^{2}} \\
& +\frac{A_{z} \frac{i \omega k}{\epsilon+p}\left(\sigma_{\epsilon}^{B}\left(1+i \omega \sigma_{\epsilon}^{B}\right)\left(\omega+i \gamma_{\eta} \boldsymbol{k}^{2}\right)-\frac{n}{\epsilon+p} \omega \sigma_{\epsilon}^{V}\left(1+i \omega \tau_{\epsilon}^{V}\right)\right)}{\left(\omega+i \gamma_{\eta} \boldsymbol{k}^{2}\right)^{2}}, \\
u^{z}= & \frac{+h_{t x} \sigma_{\epsilon}^{V}\left(1+i \omega \tau_{\epsilon}^{V}\right) i \omega k \frac{i \eta_{\eta} \boldsymbol{k}^{2}}{\epsilon+p}+h_{t z} \omega\left(\omega+i \gamma_{\eta} \boldsymbol{k}^{2}\right)+A_{z} \omega \frac{n}{\epsilon+p}\left(\omega+i \gamma_{\eta} \boldsymbol{k}^{2}\right)}{-\left(\omega+i \gamma_{\eta} \boldsymbol{k}^{2}\right)^{2}} \\
& -\frac{A_{x} \frac{i \omega k}{\epsilon+p}\left(\sigma_{\epsilon}^{B}\left(1+i \omega \sigma_{\epsilon}^{B}\right)\left(\omega+i \gamma_{\eta} \boldsymbol{k}^{2}\right)-\frac{n}{\epsilon+p} \omega \sigma_{\epsilon}^{V}\left(1+i \omega \tau_{\epsilon}^{V}\right)\right)}{\left(\omega+i \gamma_{\eta} \boldsymbol{k}^{2}\right)^{2}} .
\end{aligned}
$$

Inserting these back into the constitutive relations, we obtain the induced energy-momentum tensor and the currents that are linear in the fluctuating background $\left(h_{\mu \nu}, A_{\mu}\right)$. The retarded two-point functions of the 
energy-momenta and currents can then be obtained by differentiating these with respect to the metric and gauge field fluctuations $\left(h_{\mu \nu}, A_{\mu}\right)$.

This computation for the P-odd parts of the energy-momenta and currents retarded correlation functions is a small extension of what has already been done in Ref. [75], now including the relaxation time terms at the next leading order. Writing

$$
\begin{aligned}
\left\langle\boldsymbol{J}^{i}(k) \boldsymbol{J}^{j}(-k)\right\rangle_{R} & =i \tilde{\sigma}_{B}(k) \epsilon^{i j l} \boldsymbol{k}^{l}, & & \left\langle\boldsymbol{J}^{i}(k) T^{0 j}(-k)\right\rangle_{R}=i \tilde{\sigma}_{V}(k) \epsilon^{i j l} \boldsymbol{k}^{l}, \\
\left\langle T^{0 i}(k) \boldsymbol{J}^{j}(-k)\right\rangle_{R} & =i \tilde{\sigma}_{\epsilon}^{B}(k) \epsilon^{i j l} \boldsymbol{k}^{l}, & & \left\langle T^{0 i}(k) T^{0 j}(-k)\right\rangle_{R}=i \tilde{\sigma}_{\epsilon}^{V}(k) \epsilon^{i j l} \boldsymbol{k}^{l},
\end{aligned}
$$

we finally obtain the retarded correlation functions including the relaxation times

$$
\begin{aligned}
& \tilde{\sigma}_{B}(k)=\sigma_{B}\left(1+i \omega \tau_{B}\right)-\frac{n}{\epsilon+p}\left(\sigma_{\epsilon}^{B}\left(1+i \omega \tau_{\epsilon}^{B}\right)+\sigma_{V}\left(1+i \omega \tau_{V}\right)-\sigma_{\epsilon}^{V}\left(1+i \omega \tau_{\epsilon}^{V}\right) \frac{n}{\epsilon+p} \frac{\omega}{\omega+i \gamma_{\eta} \boldsymbol{k}^{2}}\right) \frac{\omega}{\omega+i \gamma_{\eta} \boldsymbol{k}^{2}}, \\
& \tilde{\sigma}_{V}(k)=\left(\sigma_{V}\left(1+i \omega \tau_{V}\right)-\frac{n}{\epsilon+p} \sigma_{\epsilon}^{V}\left(1+i \omega \tau_{\epsilon}^{V}\right) \frac{\omega}{\omega+i \gamma_{\eta} \boldsymbol{k}^{2}}\right) \frac{i \gamma_{\eta} \boldsymbol{k}^{2}}{\omega+i \gamma_{\eta} \boldsymbol{k}^{2}}, \\
& \tilde{\sigma}_{\epsilon}^{B}(k)=\left(\sigma_{\epsilon}^{B}\left(1+i \omega \tau_{\epsilon}^{B}\right)-\frac{n}{\epsilon+p} \sigma_{\epsilon}^{V}\left(1+i \omega \tau_{\epsilon}^{V}\right) \frac{\omega}{\omega+i \gamma_{\eta} \boldsymbol{k}^{2}}\right) \frac{i \gamma_{\eta} \boldsymbol{k}^{2}}{\omega+i \gamma_{\eta} \boldsymbol{k}^{2}}, \\
& \tilde{\sigma}_{\epsilon}^{V}(k)=\sigma_{\epsilon}^{V}\left(1+i \omega \tau_{\epsilon}^{V}\right) \frac{-\gamma_{\eta}^{2} \boldsymbol{k}^{4}}{\left(\omega+i \gamma_{\eta} \boldsymbol{k}^{2}\right)^{2}},
\end{aligned}
$$

where $\gamma_{\eta}=\eta /(\epsilon+p)$ is the shear-diffusion constant. The appearance of shear-diffusion poles in the above retarded correlation functions was explained in Ref. [75] and is due to the energy-momentum Ward identity. In the zero frequency limit, all four chiral transport coefficients decouple from each other, and these give the correct Kubo relations for the leading-order chiral transport coefficients, $\left(\sigma_{B / V}, \sigma_{\epsilon}^{B / V}\right)$. On the other hand, in the other limit of $\boldsymbol{k} \rightarrow 0$ with $\omega \neq 0$, the $\langle\boldsymbol{J} T\rangle_{R},\langle T \boldsymbol{J}\rangle_{R}$ and $\langle T T\rangle_{R}$ correlators all vanish due to the diffusion pole structure. To extract the relaxation times $\left(\tau_{B / V}, \tau_{\epsilon}^{B / V}\right)$ from these retarded correlation functions, it is clear that these diffusion pole structures must be stripped off first before taking the appropriate $\boldsymbol{k} \rightarrow 0$ limit and looking at the remaining linear term in $\omega$. For example, the Kubo relation for the relaxation time $\tau_{\epsilon}^{V}$ can be

$\tau_{\epsilon}^{V}=-\frac{1}{\sigma_{\epsilon}^{V}} \lim _{\omega \rightarrow 0} \frac{1}{\omega} \lim _{k \rightarrow 0} \operatorname{Im}\left[\frac{\left(\omega+i \gamma_{\eta} \boldsymbol{k}^{2}\right)^{2}}{-\gamma_{\eta}^{2} \boldsymbol{k}^{4}} \tilde{\sigma}_{\epsilon}^{V}(k)\right]$,

and we can write down the Kubo relations for other relaxation times:

$$
\begin{aligned}
\tau_{V}= & \frac{1}{\sigma_{V}} \lim _{\omega \rightarrow 0} \frac{1}{\omega} \lim _{\boldsymbol{k} \rightarrow 0} \operatorname{Im}\left[\frac{\omega+i \gamma_{\eta} \boldsymbol{k}^{2}}{i \gamma_{\eta} \boldsymbol{k}^{2}} \tilde{\sigma}_{V}(k)\right. \\
& \left.+\frac{n}{\epsilon+p} \sigma_{\epsilon}^{V}\left(1+i \omega \tau_{\epsilon}^{V}\right) \frac{\omega}{\omega+i \gamma_{\eta} \boldsymbol{k}^{2}}\right],
\end{aligned}
$$

and similar expressions for $\tau_{\epsilon}^{B}$ and $\tau_{B}$. Practically, we compute $\tau_{\epsilon}^{V}$ first and use it in the Kubo relations for the $\left(\tau_{V}, \tau_{\epsilon}^{B}\right)$ to compute them, and finally compute $\tau_{B}$. For these Kubo relations, we need to know $\gamma_{\eta}=\eta /(\epsilon+p)$ precisely, and fortunately in the AdS/CFT correspondence we can easily achieve this due to the Kovtun-Son-Starinets result on the shear viscosity [86], $\eta=s / 4 \pi$, that allows us to compute $\gamma_{\eta}$ precisely. Our numerical study in the AdS/CFT correspondence that will be described in more detail in Sec. IV shows that these Kubo relations can be successfully used to extract the relaxation times of chiral transport phenomena.

\section{CONSEQUENCES OF TIME-REVERSAL INVARIANCE}

In this section, we would like to derive some interesting consequences of having $\mathrm{T}$ invariance in the microscopic theory. The $\mathrm{T}$ invariance is quite generic in typical chiral (gauge) theories, since a $\mathrm{T}$ (or equivalently $C P$ ) violation could only arise from a nonremovable complex phase in the Lagrangian, which is not easy to have in general. Recall that the chirality (or helicity) that comes from a spinmomentum alignment remains invariant under $\mathrm{T}$ transformation. The ensemble with a finite chiral chemical potential does not violate $\mathrm{T}$ explicitly, since the chiral charge and chemical potential are T-even quantities. The chiral anomaly $\partial_{\mu} J^{\mu}=C E \cdot B$ is also consistent with $\mathrm{T}$ symmetry as both sides are Todd. We would like to impose 
the $\mathrm{T}$ invariance of the microscopic theory on the random noise fluctuations around equilibrium à la Onsager, whose strength is related to the spectral density via the fluctuationdissipation relation. In turn, the spectral density is obtained from transport coefficients in the hydrodynamics regime, and this gives T-invariance constraints on the transport coefficients. We will follow these steps for the P-odd thermal noise fluctuations, the P-odd spectral densities, and the chiral transport coefficients of our interests. The importance of $\mathrm{T}$ invariance in chiral transport phenomena was previously emphasized in Ref. [57].

Let us first review the general consequences of $\mathrm{T}$ invariance on the random noise fluctuations of Hermitian operators $\hat{\mathcal{O}}^{a}$ :

$$
\begin{aligned}
\left\langle\delta \mathcal{O}^{a}\left(t_{1}\right) \delta \mathcal{O}^{b}\left(t_{2}\right)\right\rangle & \equiv\left\langle\mathcal{O}_{r}^{a}\left(t_{1}\right) \mathcal{O}_{r}^{b}\left(t_{2}\right)\right\rangle \\
& =\frac{1}{Z} \operatorname{Tr}\left(e^{-\beta(\hat{H}-\mu \hat{N})} \frac{1}{2}\left\{\hat{\mathcal{O}}^{a}\left(t_{1}\right), \hat{\mathcal{O}}^{b}\left(t_{2}\right)\right\}\right) .
\end{aligned}
$$

The hatted objects are the quantum operators, and the objects without hats are the field variables in the Schwinger-Keldysh path integral. We use the " $r a$ " notation where $r=\frac{1}{2}(1+2)$ and $a=(1-2)$ with $(1,2)$ meaning the two contours of forward and backward times. Also, $Z=\operatorname{Tr}\left(e^{-\beta(\hat{H}-\mu \hat{N})}\right)$, the anticommutator is $\{\hat{A}, \hat{B}\}=$ $\hat{A} \hat{B}+\hat{B} \hat{A}$, and $\hat{\mathcal{O}}(t)=e^{i \hat{H} t} \hat{\mathcal{O}} e^{-i \hat{H} t}$. Using the Hermiticity of $\hat{\mathcal{O}}^{a}$, it is easy to see that the fluctuation correlation functions are real valued:

$$
\left\langle\mathcal{O}_{r}^{a}\left(t_{1}\right) \mathcal{O}_{r}^{b}\left(t_{2}\right)\right\rangle^{*}=\left\langle\mathcal{O}_{r}^{a}\left(t_{1}\right) \mathcal{O}_{r}^{b}\left(t_{2}\right)\right\rangle .
$$

The $\mathrm{T}$ invariance of the theory means that the Hamiltonian is invariant under complex conjugation up to a unitary transformation ${ }^{6}$ :

$$
\hat{H}^{*}=\hat{U}^{\dagger} \hat{H} \hat{U}
$$

Each physical operator is also assigned a well-defined $\mathrm{T}$ parity,

$$
\hat{U}\left(\hat{\mathcal{O}}^{a}\right)^{*} \hat{U}^{\dagger}=\tau_{a} \hat{\mathcal{O}}^{a}, \quad \tau_{a}= \pm 1
$$

For example, it is intuitively clear that the momentum density $\hat{T}^{0 i}$ and the charge currents $\hat{J}^{i}$ should have T parity $\tau=-1$, while the charge density $\hat{N}$ has T parity $\tau=+1$. From the above two equations it is easy to derive

$$
\hat{U}\left(\hat{\mathcal{O}}^{a}(t)\right)^{*} \hat{U}^{\dagger}=\tau_{a} \hat{\mathcal{O}}^{a}(-t) .
$$

\footnotetext{
${ }^{6}$ The notion of complex conjugation of an operator depends on the basis of states. This ambiguity is taken care of by additional unitary transformation $\hat{U}$.
}

By taking the complex conjugation of (3.1) and using the fact that it is real (3.2), we have

$$
\begin{aligned}
& \left\langle\mathcal{O}_{r}^{a}\left(t_{1}\right) \mathcal{O}_{r}^{b}\left(t_{2}\right)\right\rangle \\
& =\left\langle\mathcal{O}_{r}^{a}\left(t_{1}\right) \mathcal{O}_{r}^{b}\left(t_{2}\right)\right\rangle^{*} \\
& =\frac{1}{Z} \operatorname{Tr}\left(e^{-\beta\left(\hat{H}^{*}-\mu \hat{N}^{*}\right)} \frac{1}{2}\left\{\left(\hat{\mathcal{O}}^{a}\left(t_{1}\right)\right)^{*},\left(\hat{\mathcal{O}}^{b}\left(t_{2}\right)\right)^{*}\right\}\right) \\
& =\tau_{a} \tau_{b} \frac{1}{Z} \operatorname{Tr}\left(e^{-\beta(\hat{H}-\mu \hat{N})} \frac{1}{2}\left\{\hat{\mathcal{O}}^{a}\left(-t_{1}\right), \hat{\mathcal{O}}^{b}\left(-t_{2}\right)\right\}\right) \\
& =\tau_{a} \tau_{b}\left\langle\mathcal{O}_{r}^{a}\left(-t_{1}\right) \mathcal{O}_{r}^{b}\left(-t_{2}\right)\right\rangle,
\end{aligned}
$$

where we use (3.3) and (3.5) in going from the first line to the second. In terms of the frequency space correlation function defined by

$$
G_{r r}^{a b}(\omega)=\int_{-\infty}^{\infty} d t e^{i \omega t}\left\langle\mathcal{O}_{r}^{a}(t) \mathcal{O}_{r}^{b}(0)\right\rangle
$$

the $\mathrm{T}$ invariance requires that

$$
G_{r r}^{a b}(-\omega)=\tau_{a} \tau_{b} G_{r r}^{a b}(\omega)
$$

We next consider the fluctuation-dissipation relation that can be proved in general by a Lehmann-type representation:

$$
G_{r r}^{a b}(\omega)=\left(\frac{1}{2}+n_{B}(\omega)\right)\left(G_{r a}^{a b}(\omega)-G_{a r}^{a b}(\omega)\right),
$$

where the retarded correlation function is related to the " $r a$ "correlation function by $G_{R}^{a b}=-i G_{r a}^{a b}$. Up to this point, the indices $(a, b)$ could label the space position $\boldsymbol{x}$ of the operators, but let us make the space position of the operators more explicit in the following, and $(a, b)$ refer only to the operator species. We then introduce the Fourier momentum space by

$$
G_{r a}^{a b}(\omega, \boldsymbol{k}) \equiv G_{r a}^{a b}(k)=\int d^{3} \boldsymbol{x} e^{-i \boldsymbol{k} \cdot \boldsymbol{x}} G_{r a}^{a b}(\omega, \boldsymbol{x}),
$$

and the above relations (3.8) and (3.9) hold true in the Fourier momentum space as well. Note our notation $k=(\omega, \boldsymbol{k})$. The retarded correlation function in time and position coordinates can easily be shown to be real valued as they should:

$G_{R}^{a b}(t, \boldsymbol{x})=-i \theta(t) \frac{1}{Z} \operatorname{Tr}\left(e^{\beta(\hat{H}-\mu \hat{N})}\left[\hat{\mathcal{O}}^{a}(t, \boldsymbol{x}), \hat{\mathcal{O}}^{b}(0)\right]\right)$.

This implies that in the Fourier space, $G_{R}^{a b}(-k)=$ $\left(G_{R}^{a b}(k)\right)^{*}$, which is equivalent to

$$
G_{r a}^{a b}(-k)=-\left(G_{r a}^{a b}(k)\right)^{*} .
$$


From the definition, we have

$$
\begin{aligned}
G_{r a}^{a b}(t, \boldsymbol{x}) & =\left\langle\mathcal{O}_{r}^{a}(t, \boldsymbol{x}) \mathcal{O}_{a}^{b}(0)\right\rangle=\left\langle\mathcal{O}_{r}^{a}(0) \mathcal{O}_{a}^{b}(-t,-\boldsymbol{x})\right\rangle \\
& =\left\langle\mathcal{O}_{a}^{b}(-t,-\boldsymbol{x}) \mathcal{O}_{r}^{a}(0)\right\rangle=G_{a r}^{b a}(-t,-\boldsymbol{x}),
\end{aligned}
$$

where we use the translation invariance in space-time in the second equality. In the Fourier space, this translates to

$$
G_{a r}^{a b}(k)=G_{r a}^{b a}(-k)=-\left(G_{r a}^{b a}(k)\right)^{*},
$$

where the last equality comes from (3.12). Using this result, the fluctuation-dissipation relation (3.9) becomes

$$
\begin{aligned}
G_{r r}^{a b}(k) & =\left(\frac{1}{2}+n_{B}(\omega)\right)\left(G_{r a}^{a b}(k)+\left(G_{r a}^{b a}(k)\right)^{*}\right) \\
& \equiv\left(\frac{1}{2}+n_{B}(\omega)\right) \rho^{a b}(k)
\end{aligned}
$$

with the spectral density $\rho^{a b}(k)$ that is a Hermitian matrix in terms of the indices $(a, b)$. Note that the spectral density can have an imaginary part when it is antisymmetric in $(a, b)$. Indeed, this is what happens for the P-odd spectral functions arising from the P-odd retarded correlation functions of chiral transport phenomena [76]. This is consistent with the fact that the fluctuation correlation functions in space-time coordinates $G_{r r}^{a b}(t, \boldsymbol{x})$ are real valued. In terms of the retarded correlation functions, the spectral density is given by

$$
\rho^{a b}(k)=i\left(G_{R}^{a b}(k)-\left(G_{R}^{b a}(k)\right)^{*}\right) .
$$

The T-invariance constraint (3.8) and the relation (3.15) give the constraint on the spectral density. Since

$$
\left(\frac{1}{2}+n_{B}(-\omega)\right)=-\left(\frac{1}{2}+n_{B}(\omega)\right)
$$

we have the T-invariance constraint

$$
\rho^{a b}(-\omega, \boldsymbol{k})=-\tau_{a} \tau_{b} \rho^{a b}(\omega, \boldsymbol{k}),
$$

which in turn gives the constraints on the retarded correlation functions via (3.16).

We are now ready to apply this to the P-odd retarded correlation functions (2.11) that we obtained in the hydrodynamics regime in the previous section. The interesting case is when $\mathcal{O}^{a}=\boldsymbol{J}^{i}$ and $\mathcal{O}^{b}=T^{0 j}$, both of which have T parity $\tau=-1$. From (2.11) we have

$G_{R}^{a b}(k)=i \tilde{\sigma}_{V}(k) \epsilon^{i j l} \boldsymbol{k}^{l}, \quad G_{R}^{b a}(k)=-i \tilde{\sigma}_{\epsilon}^{B}(k) \epsilon^{i j l} \boldsymbol{k}^{l}$,

which gives

$$
\rho^{a b}(k)=\left(-\tilde{\sigma}_{V}(k)+\left(\tilde{\sigma}_{\epsilon}^{B}(k)\right)^{*}\right) \epsilon^{i j l} \boldsymbol{k}^{l},
$$

and we have the T-invariance constraint

$$
\tilde{\sigma}_{V}(\omega, \boldsymbol{k})-\left(\tilde{\sigma}_{\epsilon}^{B}(\omega, \boldsymbol{k})\right)^{*}=-\tilde{\sigma}_{V}(-\omega, \boldsymbol{k})+\left(\tilde{\sigma}_{\epsilon}^{B}(-\omega, \boldsymbol{k})\right)^{*} .
$$

Using the expression (2.12) of $\tilde{\sigma}_{V}(k)$ and $\tilde{\sigma}_{\epsilon}^{B}(k)$ in the hydrodynamics regime, a short algebra results in the constraint

$$
\begin{aligned}
& \left(\sigma_{V}\left(1+i \omega \tau_{V}\right)-\sigma_{\epsilon}^{B}\left(1+i \omega \tau_{\epsilon}^{B}\right)\right) \frac{1}{\omega+i \gamma_{\eta} k^{2}} \\
& \quad=\left(\sigma_{V}\left(1-i \omega \tau_{V}\right)-\sigma_{\epsilon}^{B}\left(1-i \omega \tau_{\epsilon}^{B}\right)\right) \frac{1}{\omega-i \gamma_{\eta} k^{2}},
\end{aligned}
$$

which should be satisfied for arbitrary frequency and momentum. This is true if and only if the following T-invariance constraints are met:

$$
\sigma_{V}=\sigma_{\epsilon}^{B}, \quad \tau_{V}=\tau_{\epsilon}^{B} .
$$

Our numerical computation in the AdS/CFT correspondence in the next section confirms these relations.

We can obtain a stronger version of the T-invariance constraint using rotational invariance which tells that

$$
\tilde{\sigma}_{V}(k)=\tilde{\sigma}_{V}(\omega,|\boldsymbol{k}|), \quad \tilde{\sigma}_{\epsilon}^{B}(k)=\tilde{\sigma}_{\epsilon}^{B}(\omega,|\boldsymbol{k}|) .
$$

The reality of retarded correlation functions in coordinate space dictates

$$
\tilde{\sigma}_{V}(-k)=\left(\tilde{\sigma}_{V}(k)\right)^{*}, \quad \tilde{\sigma}_{\epsilon}^{B}(-k)=\left(\tilde{\sigma}_{\epsilon}^{B}(k)\right)^{*},
$$

and together with (3.24), the T-invariance constraint (3.21) becomes

$$
\operatorname{Re}\left[\tilde{\sigma}_{V}(\omega,|\boldsymbol{k}|)\right]=\operatorname{Re}\left[\tilde{\sigma}_{\epsilon}^{B}(\omega,|\boldsymbol{k}|)\right] .
$$

The Kramers-Kronig dispersion relation of retarded correlation functions due to causality then implies that the imaginary parts of $\tilde{\sigma}_{V}(k)$ and $\tilde{\sigma}_{\epsilon}^{B}(k)$ are the same as well, and hence we finally arrive at the T-invariance constraint

$$
\tilde{\sigma}_{V}(k)=\tilde{\sigma}_{\epsilon}^{B}(k),
$$

which is valid even beyond the hydrodynamics regime.

We close this section with a short digression to Weyl semimetals. In these materials the origin of the pseudochiral massless dispersion relations for left-handed and right-handed quasiparticles are separated in the Bloch momentum space. Since the chirality is T even while the momentum flips sign under $\mathrm{T}$, this separation in momentum 
space breaks $\mathrm{T}$ invariance. It would therefore be consistent to have $\sigma_{V} \neq \sigma_{\epsilon}^{B}$ in these condensed matter systems. It seems that this is indeed the case [87]. ${ }^{7}$

\section{RELAXATION TIMES OF CHIRAL TRANSPORT PHENOMENA IN THE AdS/CFT CORRESPONDENCE}

In this section we take a minimal version of the holographic model in the AdS/CFT correspondence, where the chiral anomaly manifests as a five-dimensional ChernSimons term of the holographic gauge field that corresponds to the $U(1)_{R}$ chiral symmetry of a right-handed chiral fermion. The five-dimensional action is

$$
\begin{aligned}
S= & \frac{1}{16 \pi G} \int d^{5} x \sqrt{-g} \\
& \times\left(R+12-\frac{1}{4} F_{A B} F^{A B}+\frac{\kappa}{3} \epsilon^{M N P Q R} A_{M} F_{N P} F_{Q R}\right),
\end{aligned}
$$

where the Chern-Simons coefficient $\kappa$ should be $\kappa=$ $G /(2 \pi)$ to match the chiral anomaly for a single righthanded Weyl fermion. The $F_{M N}=\nabla_{M} A_{N}-\nabla_{N} A_{M}$ is the field strength of the $U(1)$ gauge field $A_{M}$ (capital letters run for five-dimensional coordinates, and the greek letters for four-dimensional coordinates). This theory is T invariant. ${ }^{8}$

The corresponding equations of motion are

$$
\begin{aligned}
G_{A B}-6 g_{A B}+\frac{1}{2} F_{A C} F_{B}^{C}+\frac{1}{8} g_{A B} F^{2} & =0, \\
\nabla_{B} F^{B A}+\kappa \epsilon^{A M N C D} F_{M N} F_{C D} & =0,
\end{aligned}
$$

where $g_{A B}$ is the metric, and $G_{A B}=R_{A B}-\frac{1}{2} g_{A B} R$ is the five-dimensional Einstein tensor. The equations of motion allow an exact charged black-brane solution, which describes a finite temperature plasma with non-zero chemical potential $\mu$,

$d s^{2}=r^{2}\left(-f(r) d t^{2}+d \vec{x}^{2}\right)+\frac{1}{r^{2} f(r)} d r^{2}, \quad A_{t}=-\frac{\mu r_{H}^{2}}{r^{2}}$,

where $f(r)=1-\frac{m}{r^{4}}+\frac{q^{2}}{r^{6}}$ is the blackening factor, and $r_{H}$ is the horizon where $f\left(r_{H}\right)=0$. The parameter $q$ is related to the chemical potential by $\sqrt{3} q=\mu r_{H}^{2}$, and the Hawking temperature is $T=\frac{r_{H}^{2} f^{\prime}\left(r_{H}\right)}{4 \pi}$. The equation of state of this fluid is

\footnotetext{
${ }^{7}$ We thank Karl Landsteiner for pointing this out to us.

${ }^{8}$ Under $\mathrm{T}$ transformation, the spatial components of the gauge field are odd while the time component is even, as can be inferred from their coupling to the charge current.
}

$$
p(T, \mu)=\frac{m(T, \mu)}{16 \pi G},
$$

and all other thermodynamic quantities are derived from the pressure. For our purpose, we need

$$
\begin{aligned}
\gamma_{\eta} & =\frac{\eta}{\epsilon+p}=\frac{1}{4 \pi} \frac{s}{\epsilon+p}=\frac{1}{4 \pi} \frac{(\partial p / \partial T)_{\mu}}{T(\partial p / \partial T)_{\mu}+\mu(\partial p / \partial \mu)_{T}} \\
& =\frac{1}{16 \pi} \frac{(\partial p / \partial T)_{\mu}}{p}
\end{aligned}
$$

where the last equality is from conformal nature $p=$ $T^{4} \bar{p}(T / \mu)$ or $\epsilon=3 p$.

From here on, we follow the convention and notation in Ref. [75] in our subsequent analysis. Using the scale invariance of the theory, we choose the black hole horizon radius $r_{H}$ to be unity and measure all other quantities in dimensionless units by dividing with appropriate powers of $r_{H}$. For example, the dimensionless quantities satisfy

$$
m=1+q^{2}, \quad q^{2}=\frac{\mu^{2}}{3}, \quad T=\frac{2 m-3 q^{2}}{2 \pi} .
$$

These relations allow us to characterize the plasma by a single dimensionless number, but we should keep in mind that the truly meaningful dimensionless parameter of the conformal plasma is $T / \mu$. Likewise, the meaningful value of the relaxation times in conformal theory is only given by the dimensionless number in units of inverse temperature, $\tau T$. In the holographic description, the computation of the two-point retarded correlation functions amounts to doing a variation of the on-shell action up to second order in fluctuations of the values of the gauge field and the metric at the AdS boundary $r \rightarrow \infty$. Writing the fluctuations from the above background solution as $A_{M}(r, t, \vec{x})=A_{M}^{(0)}(r)+a_{M}(r, t, \vec{x})$ and $g_{M N}(r, t, \vec{x})=$ $g_{M N}^{(0)}(r)+h_{M N}(r, t, \vec{x})$, one solves the equations of motion for the fluctuations $\left(a_{M}, h_{M N}\right)$ with the in-falling boundary condition at the horizon. The boundary values at $r \rightarrow \infty$ are fixed and they correspond to the sources coupled to the $U(1)$ current and the energy-momentum operators in the field theory side. By taking variations of the on-shell action with respect to these sources, we read off the retarded correlation functions. Since we are interested in computing the chiral transport coefficients arising from the P-odd correlation functions, it turns out to be enough to focus only on the shear sector, that is, we turn on only the following fluctuation components that are arranged in the single column vector $\Phi^{T}=\left(a_{x}, h_{t}^{x}, h_{y}^{x}, a_{z}, h_{t}^{z}, h_{y}^{z}\right)$ that only depend on $(t, y)$ coordinates. By using coordinate reparametrization invariance and gauge invariance, one can fix the gauge by $a_{r}=h_{r M}=0$. The resulting linearized equations of motion in the Fourier space of $e^{-i \omega t+i k y}$ read as 


$$
\begin{aligned}
a_{i}^{\prime \prime}(u)+\frac{f^{\prime}(u)}{f(u)} a_{i}^{\prime}(u)+\frac{1}{4 u f^{2}(u)}\left(\omega^{2}-f(u) k^{2}\right) a_{i}(u)-\frac{\mu}{f(u)} h_{t}^{\prime i}(u)+\frac{4 i k \kappa \epsilon_{i j} \mu a_{j}(u)}{f(u)} & =0, \\
h_{t}^{\prime \prime i}(u)-\frac{h_{t}^{\prime i}(u)}{u}-\frac{1}{4 u f(u)}\left(k^{2} h_{t}^{i}(u)+\omega k h_{y}^{i}(u)\right)-u \mu a_{i}^{\prime}(u) & =0, \\
h_{y}^{\prime \prime i}(u)+\left(\frac{f^{\prime}(u)}{f(u)}-\frac{1}{u}\right) h_{y}^{\prime i}(u)+\frac{1}{4 u f^{2}(u)}\left(\omega^{2} h_{y}^{i}(u)+\omega k h_{t}^{i}(u)\right) & =0,
\end{aligned}
$$

with the constraint equations

$$
\omega h^{\prime i}(u)+k f(u) h_{y}^{\prime i}(u)-u \omega \mu a_{i}(u)=0,
$$

where we use the new radial coordinate $u=\frac{1}{r^{2}}$, and $(i, j)$ runs over $x$ and $z$. Arranging the boundary sources at $u \rightarrow 0(r \rightarrow \infty)$ into a column vector

$$
\phi^{(0)}=\left\{a_{x}^{(0)}, h_{t}^{(0) x}, h_{y}^{(0) x}, a_{z}^{0}, h_{t}^{(0) z}, h_{y}^{(0) z}\right\}^{T},
$$

the solution of the equation of motion can be written in a matrix form

$$
\Phi^{I}(u)=F_{J}^{I}(k, u) \phi^{(0) J} \equiv F(k, u) \cdot \phi^{(0)},
$$

where $(I, J)$ runs over the six components of the column vectors that we introduce in the above equation.

Following Refs. [75,88], we can compute the $F(k, u)$ matrix using any set of six linearly independent solutions, $\left\{\Phi^{i}(k, u)\right\} \quad(i=1, \ldots 6)$. Note that each $\Phi^{i}(k, u)$ is a column vector. A general solution of $\Phi(u)$ is a linear combination of $\left\{\Phi^{i}(k, u)\right\}$ :

$$
\Phi(u)=\sum_{i=1}^{6} c_{i} \Phi^{i}(k, u) \equiv\left[\Phi^{i}(k, u)\right] \cdot c,
$$

where $c$ is the column vector of $c_{i}$ 's, and $\left[\Phi^{i}(k, u)\right]$ in the above expression is a matrix whose $(I, i)$ element is the $I$ th element of the column vector $\Phi^{i}(k, u)$. The constant $c_{i}$ 's are determined by matching the boundary condition at $u \rightarrow 0$ as

$$
\phi^{(0)}=\left[\Phi^{i}(k, 0)\right] \cdot c,
$$

which can be solved by

$$
c=\left(\left[\Phi^{i}(k, 0)\right]\right)^{-1} \cdot \phi^{(0)},
$$

and we have

$\Phi(u)=\left[\Phi^{i}(k, u)\right] \cdot c=\left[\Phi^{i}(k, u)\right] \cdot\left[\Phi^{i}(k, 0)\right]^{-1} \cdot \phi^{(0)}$,

which gives the $F$ matrix as

$$
F(k, u)=\left[\Phi^{i}(k, u)\right] \cdot\left[\Phi^{i}(k, 0)\right]^{-1} .
$$

In terms of the above solution of the equation of motion, the holographic renormalized action up to second order in fluctuations is given by

$$
\begin{aligned}
\delta S^{(2)}= & \int \frac{d^{4} k}{(2 \pi)^{4}}\left(\Phi^{T}(-k) \cdot A \cdot \Phi^{\prime}(k)\right. \\
& \left.+\Phi^{T}(-k) \cdot B \Phi(k)\right)\left.\right|_{u \rightarrow 0},
\end{aligned}
$$

where the derivative is along the radial direction, and the matrix $A$ is

$$
A=\frac{1}{16 \pi G} \operatorname{Diag}\left(f(u),-\frac{1}{u}, \frac{f(u)}{u}, f(u),-\frac{1}{u}, \frac{f(u)}{u}\right) .
$$

The matrix $B$ is the counterterm from the holographic renormalization, but its components vanish for our P-odd correlation functions. From these, one can read off the retarded correlation functions as

$$
G_{R}=-2 \lim _{u \rightarrow 0}\left(A \cdot(F(k, u))^{\prime}+B\right) .
$$

With all these ingredients at hand, the numerical computation of the retarded correlation functions can be done. In the zero frequency limit, the leading-order chiral transport coefficients are reproduced:

$\sigma_{B}=\frac{\kappa}{2 \pi G} \mu, \quad \sigma_{V}=\sigma_{\epsilon}^{B}=\frac{1}{2} \frac{\kappa}{2 \pi G} \mu^{2}, \quad \sigma_{\epsilon}^{V}=\frac{2}{3} \frac{\kappa}{2 \pi G} \mu^{3}$.

It is more difficult to solve the coupled second-order differential equations of motion at nonzero frequency. The system of our differential equations presents a regular singularity at $u=1$, and the Frobenius method tells us that we can isolate the singular part and the remaining has a regular series expansion around $u=1$. Out of the two possible singular parts, we select the one corresponding to the in-falling boundary condition as

$$
\begin{aligned}
& a^{i}(u)=(1-u)^{-i \omega /(4 \pi T)} b^{i}(u), \\
& h_{t}^{i}(u)=(1-u)^{-i \omega /(4 \pi T)+1} H_{t}^{i}(u), \\
& h_{y}^{i}(u)=(1-u)^{-i \omega /(4 \pi T)+1} H_{y}^{i}(u),
\end{aligned}
$$




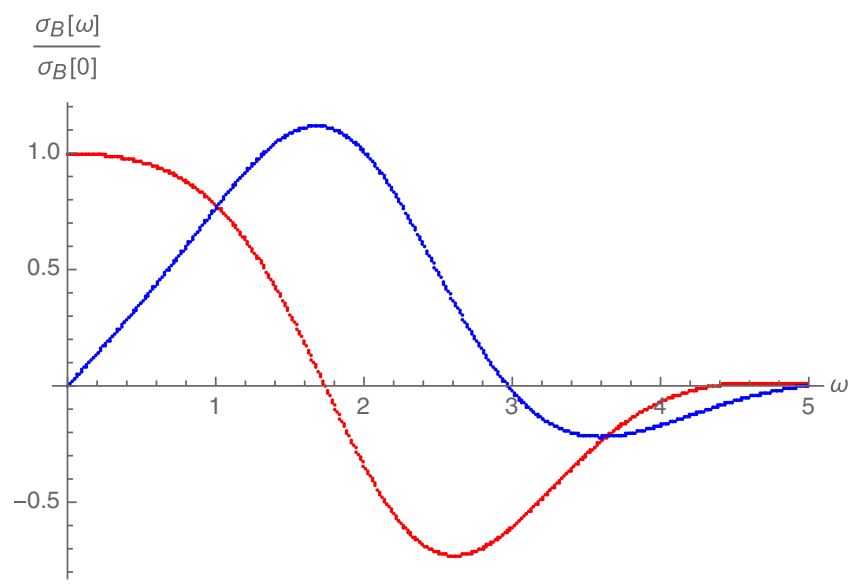

FIG. 1. The real (red) and imaginary (blue) parts of $\tilde{\sigma}_{B}(k)$ with $2 \pi T / \mu=95$ when $|\boldsymbol{k}|=0.1$ as a function of frequency.

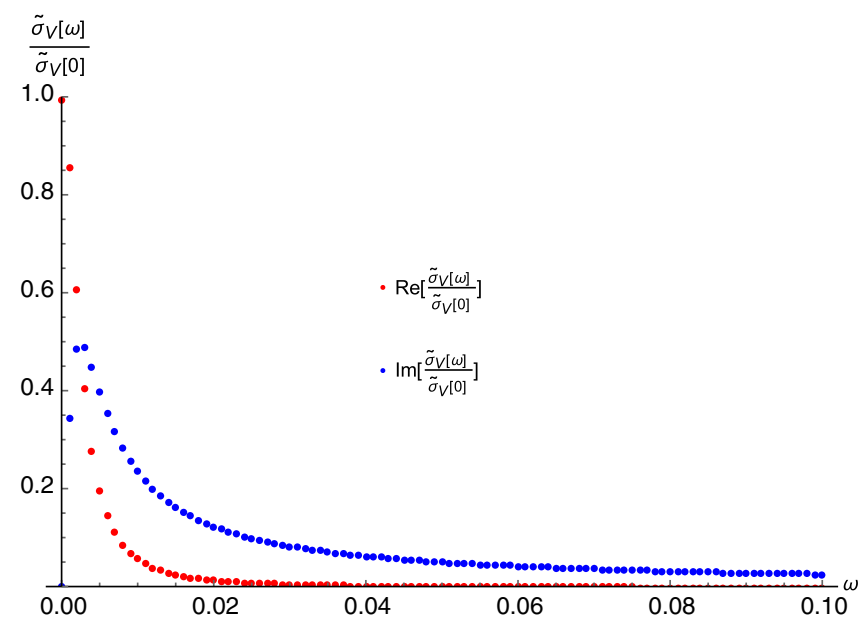

FIG. 2. The real (red) and imaginary (blue) parts of $\tilde{\sigma}_{V}(k)$ with $2 \pi T / \mu=95$ when $|\boldsymbol{k}|=0.1$ as a function of frequency.
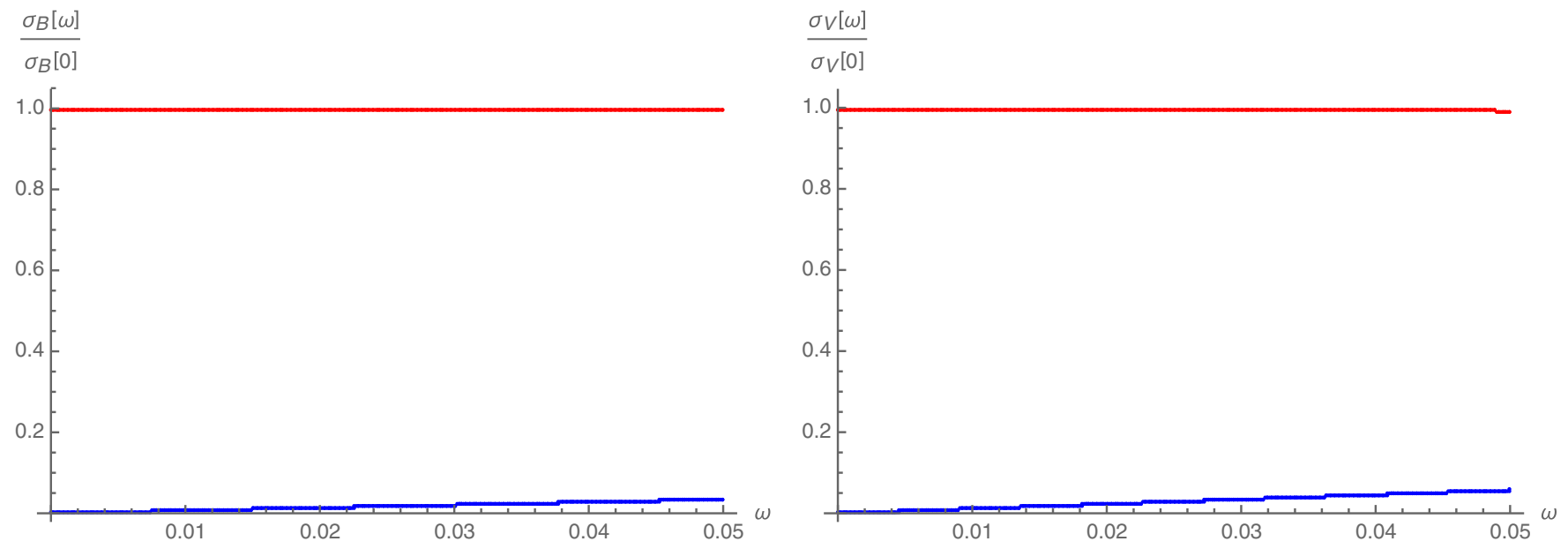

$\frac{\sigma_{\epsilon}^{B}[\omega]}{\sigma_{\epsilon}^{B}[0]}$

$\frac{\sigma_{\epsilon}^{V}[\omega]}{\sigma_{\epsilon}^{V}[0]}$
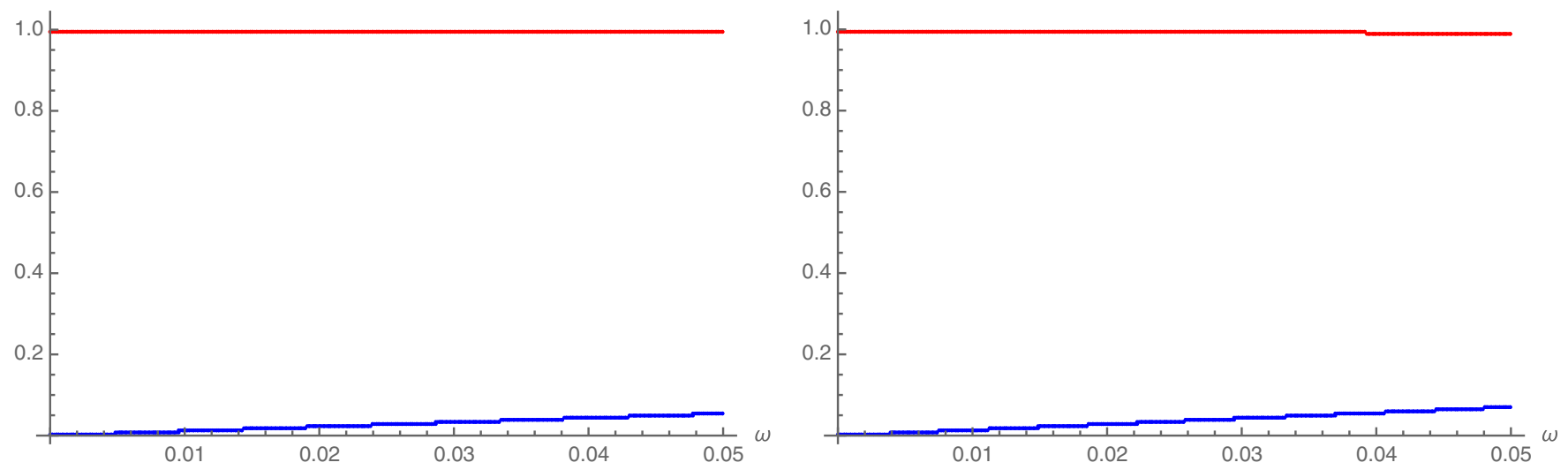

FIG. 3. The frequency-dependent chiral transport coefficients that are obtained by appropriately removing the shear-diffusion pole structures from the retarded correlation functions. The red and blue curves show the real and the imaginary parts respectively. 
where $\left(b^{i}, H_{t, y}^{i}\right)$ have regular power series expansion around $u=1$,

$$
\begin{aligned}
b^{i}(u) & =\sum_{n=0} b_{n}^{i}(1-u)^{n}, \\
H_{t}^{i}(u) & =\sum_{n=0} H_{t n}^{i}(1-u)^{n}, \\
H_{y}^{i}(u) & =\sum_{n=0} H_{y n}^{i}(1-u)^{n} .
\end{aligned}
$$

The equations of motion determine all these series coefficients in terms of the boundary values at $u=1$, that is, the six initial coefficients $\left\{b_{0}^{i}, H_{t 0}^{i}, H_{y 0}^{i}\right\}$. For example, $\left\{b^{i}(u=1)^{\prime}=-b_{1}^{i}, H_{t}^{i}(u=1)^{\prime}=-H_{t 1}^{i}, H_{y}^{i}(u=1)^{\prime}=-H_{y 1}^{i}\right\}$ are given in terms of these six initial data. This gives us six linearly independent solutions $\Phi^{i}(k, u)$ that we need. Using this information, one can start the numerical solution of the second-order differential equation slightly away from the horizon singularity $u=1+\epsilon$ with $\epsilon \ll 1$, which ensures a numerical stability. One subtlety is that we have six secondorder differential equations (4.7) and two constraints (4.8), which tells us that the six functions $\left(b^{i}, H_{t, y}^{i}\right)$ are not independent of one another. The two constraints fix the metric fluctuation $H_{y 0}^{i}$ at the horizon in terms of $b_{0}^{i}$ and $H_{t 0}^{i}$ as

$$
H_{y 0}^{i}=\frac{3(4 \pi T i+\omega)}{k\left(\mu^{2}-6\right)} H_{t 0}^{i}+\frac{12 i \pi T \mu}{k\left(\mu^{2}-6\right)} b_{0}^{i} .
$$

Due to this constraint, we can find only four linearly independent solutions. The remaining two can be trivially constructed from the gauge transformations as done in Ref. [75].

In Fig. 1 we show the numerical evaluation of the retarded correlation function $\tilde{\sigma}_{B}(k)$ with $|\boldsymbol{k}|=0.1$ as a function of frequency $\omega$ with the value of $2 \pi T / \mu=95$ corresponding to experimentally relevant values of $T=$ $160 \mathrm{MeV}$ and $\mu=10 \mathrm{MeV}$. A similar result has been previously computed in Refs. [50,75]. In Fig. 2 we plot $\tilde{\sigma}_{V}(k)$ with the same parameters. As observed previously in Ref. [75], this $\tilde{\sigma}_{V}(k)$ gets highly suppressed for small $k$ due to the shear-diffusion pole structure.

However, once we strip off the diffusion pole structure as described in Sec. II, the resulting quantity

$$
\begin{aligned}
\sigma_{V}(\omega) \equiv & \lim _{k \rightarrow 0}\left(\frac{\omega+i \gamma_{\eta} \boldsymbol{k}^{2}}{i \gamma_{\eta} \boldsymbol{k}^{2}} \tilde{\sigma}_{V}(k)\right. \\
& \left.+\frac{n}{\epsilon+p} \sigma_{\epsilon}^{V}\left(1+i \omega \tau_{\epsilon}^{V}\right) \frac{\omega}{\omega+i \gamma_{\eta} \boldsymbol{k}^{2}}\right)
\end{aligned}
$$

has the expected dependence in a small, but finite $\omega$ : in particular, the imaginary part is linear in $\omega$ with a nice finite slope that is identified as the relaxation time $\tau_{V}$.

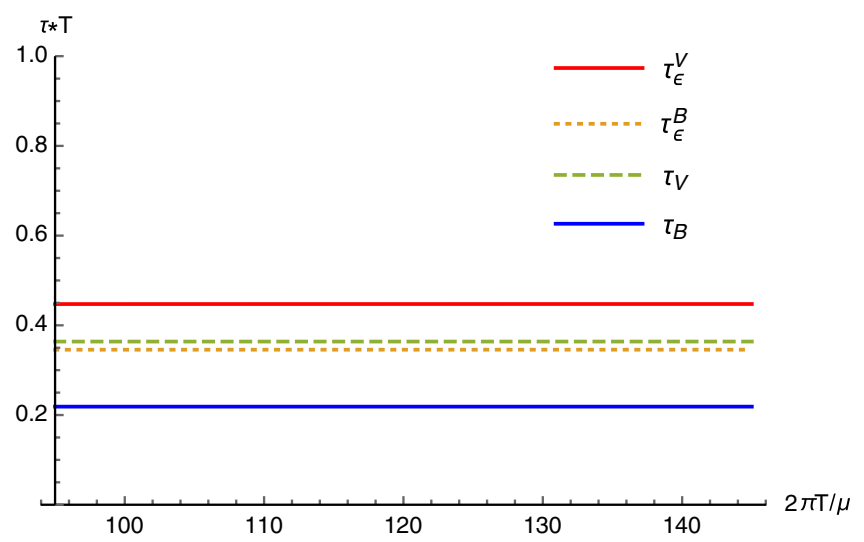

FIG. 4. The numerical result for the relaxation times of chiral transport phenomena as a function of $\tau=\frac{2 \pi T}{\mu}$.

This, together with the similar quantities $\sigma_{\epsilon}^{B / V}(\omega)$ that are obtained after stripping off the diffusion pole structures, is shown in Fig. 3. These quantities can safely be called the frequency-dependent chiral transport coefficients that appear in the constitutive relations of the chiral transport phenomena.

In Fig. 4, we plot the extracted relaxation times of the chiral transport phenomena, $\tau_{B / V}$ and $\tau_{\epsilon}^{B / V}$, as a function of the dimensionless parameter $2 \pi T / \mu$. Up to our numerical uncertainty, the result confirms our expectation of $\tau_{V}=\tau_{\epsilon}^{B}$ dictated by $\mathrm{T}$ invariance.

\section{CONCLUSION}

In this work, we compute the dynamical timescale of chiral transport phenomena, that characterizes how fast an off-equilibrium condition relaxes to the equilibrium configuration that is dictated by a chiral anomaly, in the strongly coupled regime using the AdS/CFT correspondence. The microscopic dynamics responsible for this relaxation to equilibrium is closely related to the dynamics of the spin alignment of quasiparticles in the weakly coupled regime. Although we compute these relaxation times in the strongly coupled regime using the AdS/CFT correspondence, our result should be a useful proxy for this important timescale in the real QCD plasma.

More practically, our relaxation times can be used in an Israel-Stewart-type treatment of the chiral transport phenomena in realistic hydrodynamics simulations of time-dependent background of the relativistic heavy-ion collisions. We clarify some issues related to the timedependent chiral vortical effect, by identifying correct Kubo relations for the relaxation times of chiral transport coefficients. With our Kubo relations, the relaxation times for all chiral transport phenomena, including the chiral vortical effect, are finite and well defined. This should be the case, since the underlying microscopic dynamics of the chiral vortical effect is the dynamics of spin polarization 
that must have a finite dynamical timescale to achieve the equilibrium configuration.

We also find interesting consequences of imposing timereversal invariance on the P-odd thermal noise fluctuation correlation functions, that are related to chiral transport coefficients via the fluctuation-dissipation relation. In particular we find $\tau_{V}=\tau_{\epsilon}^{B}$ which is confirmed in our numerical computation in the AdS/CFT correspondence. Our discussion also sheds light on the origin of the previously observed equality $\sigma_{V}=\sigma_{\epsilon}^{B}$ in terms of timereversal invariance.

The relaxation times that we consider in this work are examples of second-order chiral transport coefficients.
It would be interesting to compute these and the other second-order chiral transport coefficients in the weakly coupled regime in perturbative QCD, as done in Ref. [48] for $\tau_{B}$.

\section{ACKNOWLEDGMENTS}

We thank Dima Kharzeev, Karl Landsteiner, Misha Stephanov and Yi Yin for helpful discussions. This work is supported by the U.S. Department of Energy, Office of Science, Office of Nuclear Physics, with Grant No. DESC0018209, and within the framework of the Beam Energy Scan Theory (BEST) Topical Collaboration.
[1] D. E. Kharzeev, The chiral magnetic effect and anomalyinduced transport, Prog. Part. Nucl. Phys. 75, 133 (2014).

[2] D. E. Kharzeev, J. Liao, S. A. Voloshin, and G. Wang, Chiral magnetic and vortical effects in high-energy nuclear collisions-status report, Prog. Part. Nucl. Phys. 88, 1 (2016).

[3] V. Koch, S. Schlichting, V. Skokov, P. Sorensen, J. Thomas, S. Voloshin, G. Wang, and H. U. Yee, Status of the chiral magnetic effect and collisions of isobars, Chin. Phys. C 41, 072001 (2017).

[4] D. E. Kharzeev, K. Landsteiner, A. Schmitt, and H. U. Yee, Strongly interacting matter in magnetic fields: An overview, Lect. Notes Phys. 871, 1 (2013).

[5] V. A. Miransky and I. A. Shovkovy, Quantum field theory in a magnetic field: From quantum chromodynamics to graphene and Dirac semimetals, Phys. Rep. 576, 1 (2015).

[6] K. Hattori and X. G. Huang, Novel quantum phenomena induced by strong magnetic fields in heavy-ion collisions, Nucl. Sci. Tech. 28, 26 (2017).

[7] K. Fukushima, D. E. Kharzeev, and H. J. Warringa, The chiral magnetic effect, Phys. Rev. D 78, 074033 (2008).

[8] A. Vilenkin, Equilibrium parity violating current in a magnetic field, Phys. Rev. D 22, 3080 (1980).

[9] D. Kharzeev and A. Zhitnitsky, Charge separation induced by P-odd bubbles in QCD matter, Nucl. Phys. A797, 67 (2007).

[10] D. E. Kharzeev, L. D. McLerran, and H. J. Warringa, The effects of topological charge change in heavy ion collisions: Event by event $\mathrm{P}$ and $C P$ violation, Nucl. Phys. A803, 227 (2008).

[11] Y. Hirono, T. Hirano, and D. E. Kharzeev, The chiral magnetic effect in heavy-ion collisions from event-by-event anomalous hydrodynamics, arXiv:1412.0311.

[12] Y. Jiang, S. Shi, Y. Yin, and J. Liao, Quantifying the chiral magnetic effect from anomalous-viscous fluid dynamics, Chin. Phys. C 42, 011001 (2018).

[13] V. Skokov, A. Y. Illarionov, and V. Toneev, Estimate of the magnetic field strength in heavy-ion collisions, Int. J. Mod. Phys. A 24, 5925 (2009).
[14] A. Bzdak and V. Skokov, Event-by-event fluctuations of magnetic and electric fields in heavy ion collisions, Phys. Lett. B 710, 171 (2012).

[15] W. T. Deng and X. G. Huang, Event-by-event generation of electromagnetic fields in heavy-ion collisions, Phys. Rev. C 85, 044907 (2012).

[16] J. Bloczynski, X. G. Huang, X. Zhang, and J. Liao, Azimuthally fluctuating magnetic field and its impacts on observables in heavy-ion collisions, Phys. Lett. B 718, 1529 (2013).

[17] K. Tuchin, Electromagnetic field and the chiral magnetic effect in the quark-gluon plasma, Phys. Rev. C 91, 064902 (2015).

[18] L. McLerran and V. Skokov, Comments about the electromagnetic field in heavy-ion collisions, Nucl. Phys. A929, 184 (2014).

[19] S. A. Voloshin, Parity violation in hot QCD: How to detect it, Phys. Rev. C 70, 057901 (2004).

[20] B. I. Abelev et al. (STAR Collaboration), Azimuthal Charged-Particle Correlations and Possible Local Strong Parity Violation, Phys. Rev. Lett. 103, 251601 (2009).

[21] B. I. Abelev et al. (STAR Collaboration), Observation of charge-dependent azimuthal correlations and possible local strong parity violation in heavy ion collisions, Phys. Rev. C 81, 054908 (2010).

[22] L. Adamczyk et al. (STAR Collaboration), Observation of Charge Asymmetry Dependence of Pion Elliptic Flow and the Possible Chiral Magnetic Wave in Heavy-Ion Collisions, Phys. Rev. Lett. 114, 252302 (2015).

[23] J. Adam et al. (ALICE Collaboration), Charge-dependent flow and the search for the chiral magnetic wave in $\mathrm{Pb}-\mathrm{Pb}$ collisions at $\sqrt{s_{\mathrm{NN}}}=2.76 \mathrm{TeV}$, Phys. Rev. C 93, 044903 (2016).

[24] J. Erdmenger, M. Haack, M. Kaminski, and A. Yarom, Fluid dynamics of R-charged black holes, J. High Energy Phys. 01 (2009) 055.

[25] N. Banerjee, J. Bhattacharya, S. Bhattacharyya, S. Dutta, R. Loganayagam, and P. Surowka, Hydrodynamics from charged black branes, J. High Energy Phys. 01 (2011) 094. 
[26] D. E. Kharzeev and D. T. Son, Testing the Chiral Magnetic and Chiral Vortical Effects in Heavy Ion Collisions, Phys. Rev. Lett. 106, 062301 (2011).

[27] Y. Jiang, X. G. Huang, and J. Liao, Chiral vortical wave and induced flavor charge transport in a rotating quark-gluon plasma, Phys. Rev. D 92, 071501 (2015).

[28] D. Kharzeev, A. Krasnitz, and R. Venugopalan, Anomalous chirality fluctuations in the initial stage of heavy ion collisions and parity odd bubbles, Phys. Lett. B 545, 298 (2002).

[29] M. Mace, S. Schlichting, and R. Venugopalan, Offequilibrium sphaleron transitions in the glasma, Phys. Rev. D 93, 074036 (2016).

[30] M. Mace, N. Mueller, S. Schlichting, and S. Sharma, Nonequilibrium study of the chiral magnetic effect from realtime simulations with dynamical fermions, Phys. Rev. D 95, 036023 (2017).

[31] D. E. Kharzeev and H.-U. Yee, Chiral magnetic wave, Phys. Rev. D 83, 085007 (2011).

[32] G. M. Newman, Anomalous hydrodynamics, J. High Energy Phys. 01 (2006) 158.

[33] Y. Burnier, D. E. Kharzeev, J. Liao, and H. U. Yee, Chiral Magnetic Wave at Finite Baryon Density and the Electric Quadrupole Moment of Quark-Gluon Plasma in Heavy Ion Collisions, Phys. Rev. Lett. 107, 052303 (2011).

[34] E. V. Gorbar, V. A. Miransky, and I. A. Shovkovy, Normal ground state of dense relativistic matter in a magnetic field, Phys. Rev. D 83, 085003 (2011).

[35] H. U. Yee and Y. Yin, Realistic implementation of chiral magnetic wave in heavy ion collisions, Phys. Rev. C 89, 044909 (2014).

[36] M. Asakawa, A. Majumder, and B. Muller, Electric charge separation in strong transient magnetic fields, Phys. Rev. C 81, 064912 (2010).

[37] A. Bzdak, V. Koch, and J. Liao, Remarks on possible local parity violation in heavy ion collisions, Phys. Rev. C 81, 031901 (2010).

[38] F. Wang, Effects of Cluster particle correlations on local parity violation observables, Phys. Rev. C 81, 064902 (2010).

[39] S. Pratt, S. Schlichting, and S. Gavin, Effects of momentum conservation and flow on angular correlations at RHIC, Phys. Rev. C 84, 024909 (2011).

[40] A. Bzdak and P. Bozek, Contributions to the event-by-event charge asymmetry dependence for the elliptic flow of $p i^{+}$ and $p i^{-}$in heavy-ion collisions, Phys. Lett. B 726, 239 (2013).

[41] W. T. Deng, X. G. Huang, G. L. Ma, and G. Wang, Test the chiral magnetic effect with isobaric collisions, Phys. Rev. C 94, 041901 (2016).

[42] Q. Li, D. E. Kharzeev, C. Zhang, Y. Huang, I. Pletikosić, A. V. Fedorov, R. D. Zhong, J. A. Schneeloch, G. D. Gu, and T. Valla, Observation of the chiral magnetic effect in ZrTe5, Nat. Phys. 12, 550 (2016).

[43] X. Huang et al., Observation of the Chiral-AnomalyInduced Negative Magnetoresistance in 3D Weyl Semimetal TaAs, Phys. Rev. X 5, 031023 (2015).

[44] C. Zhang et al., Signatures of the Adler-Bell-Jackiw chiral anomaly in a Weyl fermion semimetal, Nat. Commun. 7, 10735 (2016).
[45] K. Landsteiner, E. Megias, and F. Pena-Benitez, Gravitational Anomaly and Transport, Phys. Rev. Lett. 107, 021601 (2011).

[46] K. Landsteiner, E. Megias, L. Melgar, and F. Pena-Benitez, Holographic gravitational anomaly and chiral vortical effect, J. High Energy Phys. 09 (2011) 121.

[47] D. E. Kharzeev and H. J. Warringa, Chiral magnetic conductivity, Phys. Rev. D 80, 034028 (2009).

[48] A. Jimenez-Alba and H. U. Yee, Second order transport coefficient from the chiral anomaly at weak coupling: Diagrammatic resummation, Phys. Rev. D 92, 014023 (2015).

[49] D. Satow and H. U. Yee, Chiral magnetic effect at weak coupling with relaxation dynamics, Phys. Rev. D 90, 014027 (2014).

[50] H. U. Yee, Holographic chiral magnetic conductivity, J. High Energy Phys. 11 (2009) 085.

[51] A. Rebhan, A. Schmitt, and S. A. Stricker, Anomalies and the chiral magnetic effect in the Sakai-Sugimoto model, J. High Energy Phys. 01 (2010) 026.

[52] A. Gynther, K. Landsteiner, F. Pena-Benitez, and A. Rebhan, Holographic anomalous conductivities and the chiral magnetic effect, J. High Energy Phys. 02 (2011) 110.

[53] C. Hoyos, T. Nishioka, and A. O'Bannon, A chiral magnetic effect from AdS/CFT with flavor, J. High Energy Phys. 10 (2011) 084.

[54] Y. Bu, M. Lublinsky, and A. Sharon, Anomalous transport from holography: Part I, J. High Energy Phys. 11 (2016) 093.

[55] M. Ammon, M. Kaminski, R. Koirala, J. Leiber, and J. Wu, Quasinormal modes of charged magnetic black branes and chiral magnetic transport, J. High Energy Phys. 04 (2017) 067.

[56] D. T. Son and P. Surowka, Hydrodynamics with Triangle Anomalies, Phys. Rev. Lett. 103, 191601 (2009).

[57] D. E. Kharzeev and H. U. Yee, Anomalies and time reversal invariance in relativistic hydrodynamics: The second order and higher dimensional formulations, Phys. Rev. D 84, 045025 (2011).

[58] R. Loganayagam, Anomaly Induced transport in arbitrary dimensions, arXiv:1106.0277.

[59] K. Jensen, R. Loganayagam, and A. Yarom, Thermodynamics, gravitational anomalies and cones, J. High Energy Phys. 02 (2013) 088.

[60] K. Jensen, R. Loganayagam, and A. Yarom, Chern-Simons terms from thermal circles and anomalies, J. High Energy Phys. 05 (2014) 110.

[61] A. V. Sadofyev, V. I. Shevchenko, and V. I. Zakharov, Notes on chiral hydrodynamics within effective theory approach, Phys. Rev. D 83, 105025 (2011).

[62] D. T. Son and N. Yamamoto, Berry Curvature, Triangle Anomalies, and the Chiral Magnetic Effect in Fermi Liquids, Phys. Rev. Lett. 109, 181602 (2012).

[63] M. A. Stephanov and Y. Yin, Chiral Kinetic Theory, Phys. Rev. Lett. 109, 162001 (2012).

[64] J. W. Chen, S. Pu, Q. Wang, and X. N. Wang, Berry Curvature and Four-Dimensional Monopoles in the Relativistic Chiral Kinetic Equation, Phys. Rev. Lett. 110, 262301 (2013). 
[65] D. T. Son and N. Yamamoto, Kinetic theory with Berry curvature from quantum field theories, Phys. Rev. D 87, 085016 (2013).

[66] J. Y. Chen, D. T. Son, M. A. Stephanov, H. U. Yee, and Y. Yin, Lorentz Invariance in Chiral Kinetic Theory, Phys. Rev. Lett. 113, 182302 (2014).

[67] C. Manuel and J. M. Torres-Rincon, Chiral transport equation from the quantum Dirac Hamiltonian and the on-shell effective field theory, Phys. Rev. D 90, 076007 (2014).

[68] J. Y. Chen, D. T. Son, and M. A. Stephanov, Collisions in Chiral Kinetic Theory, Phys. Rev. Lett. 115, 021601 (2015).

[69] N. Mueller and R. Venugopalan, Worldline construction of a covariant chiral kinetic theory, Phys. Rev. D 96, 016023 (2017).

[70] Y. Hidaka, S. Pu, and D. L. Yang, Relativistic chiral kinetic theory from quantum field theories, Phys. Rev. D 95, 091901 (2017).

[71] A. Huang, S. Shi, Y. Jiang, J. Liao, and P. Zhuang, Complete and consistent chiral transport from Wigner function formalism, Phys. Rev. D 98, 036010 (2018).

[72] C. Duval and P. A. Horvathy, Chiral fermions as classical massless spinning particles, Phys. Rev. D 91, 045013 (2015).

[73] D. E. Kharzeev, M. A. Stephanov, and H. U. Yee, Anatomy of chiral magnetic effect in and out of equilibrium, Phys. Rev. D 95, 051901 (2017).

[74] I. Amado, K. Landsteiner, and F. Pena-Benitez, Anomalous transport coefficients from Kubo formulas in holography, J. High Energy Phys. 05 (2011) 081.

[75] K. Landsteiner, E. Megias, and F. Pena-Benitez, Frequency dependence of the chiral vortical effect, Phys. Rev. D 90, 065026 (2014).

[76] K. A. Mamo and H. U. Yee, Spin polarized photons from an axially charged plasma at weak coupling: Complete leading order, Phys. Rev. D 93, 065053 (2016).
[77] E. Megias and M. Valle, Second-order partition function of a non-interacting chiral fluid in $3+1$ dimensions, J. High Energy Phys. 11 (2014) 005.

[78] G. S. Denicol, J. Noronha, H. Niemi, and D. H. Rischke, Origin of the relaxation time in dissipative fluid dynamics, Phys. Rev. D 83, 074019 (2011).

[79] R. Baier, P. Romatschke, D. T. Son, A. O. Starinets, and M. A. Stephanov, Relativistic viscous hydrodynamics, conformal invariance, and holography, J. High Energy Phys. 04 (2008) 100.

[80] R. Loganayagam and P. Surowka, Anomaly/transport in an ideal Weyl gas, J. High Energy Phys. 04 (2012) 097.

[81] K. Landsteiner, E. Megias, and F. Pena-Benitez, Anomalous transport from Kubo formulae, Lect. Notes Phys. 871, 433 (2013).

[82] M. A. Stephanov and H. U. Yee, No-Drag Frame for Anomalous Chiral Fluid, Phys. Rev. Lett. 116, 122302 (2016).

[83] K. Rajagopal and A. V. Sadofyev, Chiral drag force, J. High Energy Phys. 10 (2015) 018.

[84] L. Adamczyk et al. (STAR Collaboration), Global $\Lambda$ hyperon polarization in nuclear collisions: Evidence for the most vortical fluid, Nature (London) 548, 62 (2017).

[85] H. U. Yee, Chiral Magnetic and vortical effects in higher dimensions at weak coupling, Phys. Rev. D 90, 065021 (2014).

[86] P. Kovtun, D. T. Son, and A. O. Starinets, Viscosity in Strongly Interacting Quantum Field Theories from Black Hole Physics, Phys. Rev. Lett. 94, 111601 (2005).

[87] K. Landsteiner, Anomalous transport of Weyl fermions in Weyl semimetals, Phys. Rev. B 89, 075124 (2014).

[88] M. Kaminski, K. Landsteiner, F. Pena-Benitez, J. Erdmenger, C. Greubel, and P. Kerner, Quasinormal modes of massive charged flavor branes, J. High Energy Phys. 03 (2010) 117. 